\title{
On the Irreducibility of Wada's Representation of the Pure Braid Group, $P_{4}$
}

\author{
Ghenwa H. Abboud* and Mohammad N. Abdulrahim*
}

Department of Mathematics, Beirut Arab University, P.O. Box 11-5020, Beirut, Lebanon

\begin{abstract}
We consider the reduced Wada's representation of the pure braid group, namely $P_{4} \rightarrow G L_{3}\left(\mathbb{Z}\left[t_{1}^{ \pm 1}, t_{2}^{ \pm 1}, t_{3}^{ \pm 1}, t_{4}^{ \pm 1}\right]\right)$. We then specialize the parameters $t_{1}, t_{2}, t_{3}, t_{4}$ to nonzero complex numbers $z_{1}, z_{2}, z_{3}, z_{4}$. Our main theorem asserts that the reduced Wada's representation, $\varphi_{4}: P_{4} \rightarrow G L_{3}(\mathbb{C})$, is reducible if and only if $z_{1}^{2}=z_{2}^{2}=z_{3}^{2}=z_{4}^{2}$.
\end{abstract}

Keywords: Pure braid group, Wada's representation,irreducible.

\section{INTRODUCTION}

Let $B_{n}$ be the braid group on $n$ strings. We consider a normal subgroup, namely the pure braid group, denoted by $P_{n}$. In section 2, we define Wada's representation of pure braid group on four strings. Under that representation, the automorphism corresponding to $\sigma_{i}$, takes $x_{i} \rightarrow x_{i} x_{i+1}^{-1} x_{i}$, $x_{i+1} \rightarrow x_{i}$; and fixes all other free generators. We then specialize the indeterminates used in defining the representation $P_{4} \rightarrow G L_{4}\left(\mathbb{Z}\left[t_{1}^{ \pm 1}, \ldots, t_{4}^{ \pm 1}\right]\right)$ to nonzero complex numbers $a, b, c$ and $d$. In [1], it was shown that the reduced Wada's representation $B_{n} \rightarrow G L_{n-1}(\mathbb{C})$ is irreducible if and only if $n$ is an odd integer. In section 3 , we consider the question of the irreducibility after we restrict the representation to the normal subgroup of $B_{4}$, namely the pure braid group $P_{4}$. In other words, we determine necessary and sufficent conditions under which $\varphi_{4}(a, b, c, d): P_{4} \rightarrow G L_{3}(\mathbb{C})$ is reducible.

\section{DEFINITIONS}

\section{Definition 1}

The braid group on $n$ strings, $B_{n}$, is the abstract group with presentation

$$
\begin{aligned}
& B_{n}=\left\{\sigma_{1} \ldots, \sigma_{n-1} / \sigma_{i} \sigma_{i+1} \sigma_{i}=\sigma_{i+1} \sigma_{i} \sigma_{i+1} \text { fori }=1, \ldots, n-2, \sigma_{i} \sigma_{j}\right. \\
& \left.=\sigma_{j} \sigma_{i} \text { if }|i-j|>1\right\} .
\end{aligned}
$$

The generators $\sigma_{1}, \sigma_{2}, \ldots, \sigma_{n-1}$ are called the standard generators of $B_{n}$ (See [3]).

*Address correspondence to these authors at the Department of Mathematics, Beirut Arab University, P.O. Box 11-5020, Beirut, Lebanon; Tel: 9617-985858; Fax: 9611-818402; E-mails: gha009@bau.edu.lb; mna@bau.edu.lb

\section{Definition 2}

The pure braid group, denoted by $P_{n}$, is defined as the kernel of the homomorphism $B_{n} \rightarrow S_{n}$ defined by $\sigma_{i} \rightarrow(i$, $i+1), \quad 1 \leq i \leq n-1$ (See [2]). It is finitely generated by the elements

$A_{i j}=\sigma_{j-1} \sigma_{j-2} \ldots \sigma_{i+1} \sigma_{i}^{2} \sigma_{i+1}^{-1} \ldots \sigma_{j-2}^{-1} \sigma_{j-1}^{-1}, 1 \leq i<j \leq n$.

Let $F_{n}$ be the free group of rank $n$, with free basis $x_{1}, \ldots, x_{n}$. According to Wada's representation, the action of braid generators $\sigma_{i}$ on the basis $\left\{x_{1}, \ldots, x_{n}\right\}$ is defined as follows:

$$
\sigma_{\mathrm{i}}:\left\{\begin{array}{l}
\mathrm{x}_{\mathrm{i}} \rightarrow \mathrm{x}_{\mathrm{i}} \mathrm{x}_{\mathrm{i}+1}^{-1} \mathrm{x}_{\mathrm{i}} \\
\mathrm{x}_{\mathrm{i}+1} \rightarrow \mathrm{x}_{\mathrm{i}} \\
\mathrm{x}_{\mathrm{j}} \rightarrow \mathrm{x}_{\mathrm{j}} \text { forj } \notin\{\mathrm{i}, \mathrm{i}+1\}
\end{array}\right.
$$

By applying the Magnus representation to the image of the pure braid group under Wada' $s$ representation, we determine the linear representation $P_{4} \rightarrow G L_{4}\left(\mathbb{Z}\left[t_{1}^{ \pm 1}, \ldots, t_{4}^{ \pm 1}\right]\right)$. Now we specialize the indeterminantes $t_{1}, \ldots, t_{4}$ in Wada's representation to nonzero complex numbers $\mathrm{a}, \mathrm{b}, \mathrm{c}$ and $\mathrm{d}$ respectively. We then conjugate this representation by a matrix $\mathrm{T}$ defined by

$\mathrm{T}=\left(\begin{array}{llll}1 & 1 & 0 & 0 \\ 1 & 0 & 0 & 0 \\ 1 & 0 & 1 & 0 \\ 1 & 0 & 0 & 1\end{array}\right)$.

Having done this, we observe that entries $(2,1),(3,1)$ and $(4,1)$ of the images of all the generators of $P_{4}$ under Wada's representation are zeros. Therefore, we may delete the first row and the first column to obtain a representation 
of degree 3 , and we denote the representation by $\varphi_{4}$. For simplicity, we still call $T^{-1} A_{i j} T$ by $A_{i j}$ for $1 \leq i<j \leq 4$.

\section{Definition 3}

For $(a, b, c, d) \in\left(\mathbb{C}^{*}\right)^{4}$, the reduced Wada's representation $\varphi_{4}(a, b, c, d): P_{4} \rightarrow G L_{3}(\mathbb{C})$ is given by

$$
A_{12}=\left(\begin{array}{ccc}
\frac{a^{2}}{b^{2}} & 0 & 0 \\
-\frac{a+b}{b} & 1 & 0 \\
-\frac{a+b}{b} & 0 & 1
\end{array}\right), A_{13}=\left(\begin{array}{ccc}
1+\frac{a c\left(b^{2}+a c\right)}{b^{4}} & \frac{a\left(b^{2}+a c\right)}{b^{3}} & 0 \\
-\frac{b\left(b^{2}+a c\right)}{a^{2} c} & \frac{-b^{2}}{a c} & 0 \\
0 & 0 & 1
\end{array}\right),
$$$$
\mathrm{A}_{23}=\left(\begin{array}{ccc}
1 & \frac{\mathrm{b}(\mathrm{b}+\mathrm{c})}{\mathrm{c}^{2}} & 0 \\
0 & \frac{\mathrm{b}^{2}}{\mathrm{c}^{2}} & 0 \\
0 & \frac{\mathrm{b}(\mathrm{b}+\mathrm{c})}{\mathrm{c}^{2}} & 1
\end{array}\right), \mathrm{A}_{34}=\left(\begin{array}{ccc}
1 & 0 & 0 \\
0 & \frac{\mathrm{c}^{2}+\mathrm{cd}+\mathrm{d}^{2}}{\mathrm{~d}^{2}} & \frac{-\mathrm{c}(\mathrm{c}+\mathrm{d})}{\mathrm{d}^{2}} \\
0 & \frac{\mathrm{c}+\mathrm{d}}{\mathrm{d}} & -\frac{\mathrm{c}}{\mathrm{d}}
\end{array}\right)
$$$$
A_{14}=\left(\begin{array}{ccc}
1+\frac{a c^{2}\left(a c^{2}+b^{2} d\right)}{b^{4} d^{2}} & \frac{a(c+d)\left(a c^{2}+b^{2} d\right)}{b^{3} d^{2}} & \frac{-a c\left(a c^{2}+b^{2} d\right)}{b^{3} d^{2}} \\
0 & 1 & \frac{-c(c+d)}{d^{2}} \\
\frac{c\left(b^{2} d+a c^{2}\right)}{b^{3} d} & \frac{\left(b^{2}+a c\right)\left(a c^{2}+b^{2} d\right)}{b^{4} d} & -\frac{c}{d}
\end{array}\right)
$$

and

$$
\mathrm{A}_{24}=\left(\begin{array}{ccc}
1 & \frac{b(c+d)\left(c^{2}+b d\right)}{c^{4}} & -\frac{b\left(c^{2}+b d\right)}{c^{3}} \\
0 & \frac{c^{4}+b\left(c^{2}+b d\right)(c+d)}{c^{4}} & \frac{-b\left(c^{2}+b d\right)}{c^{3}} \\
0 & \frac{\left(c^{2}+b d\right)\left(b c^{4}+c^{5}+b^{3} d c+b^{3} d^{2}\right)}{b^{2} c^{4} d} & -\frac{\left(c^{5}+b^{2} c^{2} d+b^{3} d^{2}\right)}{b c^{3} d}
\end{array}\right) .
$$

\section{IRREDUCIBILITY OF $\phi_{4}$}

We determine necessary and sufficient conditions under which the complex specialization $\phi_{4}(\mathrm{a}, \mathrm{b}, \mathrm{c}, \mathrm{d})$ is irreducible.

\section{Lemma 4}

For $(a, b, c, d) \in\left(\mathbb{C}^{*}\right)^{4}$, the reduced Wada's represntation $\phi_{4}(\mathrm{a}, \mathrm{b}, \mathrm{c}, \mathrm{d}): \mathrm{P}_{4} \rightarrow \mathrm{GL}_{3}(\mathbb{C})$ is irreducible if $\mathrm{a}^{2} \neq \mathrm{b}^{2}$ or $\mathrm{b}^{2} \neq \mathrm{c}^{2}$ or $\mathrm{c}^{2} \neq \mathrm{d}^{2}$.

Proof. Let $\mathrm{a}^{2} \neq \mathrm{b}^{2}$. We diagonalize the matrix that corresponds to the pure braid $\mathrm{A}_{12}$ by an invertible matrix $M$ defined by

$$
M=\left(\begin{array}{ccc}
0 & 0 & 1-\mathrm{ab}^{-1} \\
0 & 1 & 1 \\
1 & 0 & 1
\end{array}\right)
$$

Direct computations show that
$M^{-1} A_{12} M=\left(\begin{array}{ccc}1 & 0 & 0 \\ 0 & 1 & 0 \\ 0 & 0 & a^{2} b^{-2}\end{array}\right)$.

Now we conjugate the reduced Wada's representation, $\varphi_{4}$, by $M$ to get an equivalent representation of degree 3 . For simplicity, we still denote $M^{-1} A_{i j} M$ by $A_{i j}$ for $1 \leq i<j \leq 4$. The matrices are given by

$$
\begin{aligned}
& A_{24}=\left(\begin{array}{ccc}
\frac{-c^{2}}{b d}-\frac{a b n}{(a-b) c^{3}} & \frac{p n}{(a-b) b^{2} c^{4} d} & \frac{n c}{b^{2} d}+\frac{a b d n}{(a-b) c^{4}} \\
\frac{a b n}{(-a+b) c^{3}} & \frac{q}{(a-b) c^{4}} & \frac{a b d n}{(a-b) c^{4}} \\
\frac{b^{2} n}{(a-b) c^{3}} & \frac{-b^{2} n(c+d)}{(a-b) c^{4}} & 1-\frac{b^{2} d n}{(a-b) c^{4}}
\end{array}\right) \\
& A_{13}=\left(\begin{array}{ccc}
1 & \frac{a\left(b^{2}+a c\right)}{(a-b) b^{2}} & \frac{-a\left(b^{2}+a c\right)\left(-b^{2}+a c-b c\right)}{(a-b) b^{4}} \\
0 & \frac{-b^{2}}{a c}+\frac{a\left(b^{2}+a c\right)}{(a-b) b^{2}} & \frac{-b\left(b^{2}+a c\right)}{a^{2} c}+\frac{a\left(b^{2}+a c\right)(b(b+c)+a c)}{(a-b) b^{4}} \\
0 & \frac{-a\left(b^{2}+a c\right)}{(a-b) b^{2}} & \frac{-b^{5}-a b^{3} c+a^{3} c^{2}-a^{2} b c^{2}}{(a-b) b^{4}}
\end{array}\right), \\
& A_{14}=\left(\begin{array}{ccc}
\frac{a c m}{(b-a) b^{2} d^{2}}-\frac{a c^{2}}{b^{2} d} & \frac{m\left(b^{2}+a c\right)}{b^{4} d}+\frac{m a(c+d)}{b^{2} d^{2}(a-b)} & \frac{m c}{b^{3} d}+\frac{a m\left(b n-a c^{2}\right)}{b^{4} d^{2}(a-b)} \\
\frac{-a c m}{(a-b) b^{2} d^{2}} & 1+\frac{a m(c+d)}{(a-b) b^{2} d^{2}} & \frac{-a m c^{2}}{b^{4} d^{2}}+\frac{a m}{(a-b) b^{2} d} \\
\frac{a c m}{(a-b) b^{2} d^{2}} & \frac{-a m(c+d)}{(a-b) b^{2} d^{2}} & \frac{a^{2} c^{4}}{b^{4} d^{2}}-\frac{m}{(a-b) b d}
\end{array}\right), \\
& A_{34}=\left(\begin{array}{ccc}
\frac{-c}{d} & \frac{c+d}{d} & 0 \\
\frac{-c(c+d)}{d^{2}} & \frac{c^{2}+c d+d^{2}}{d^{2}} & 0 \\
0 & 0 & 1
\end{array}\right) \text {, } \\
& A_{23}=\left(\begin{array}{ccc}
1 & \frac{-a b(b+c)}{(-a+b) c^{2}} & \frac{a b(b+c)}{(a-b) c^{2}} \\
0 & \frac{-b^{2}(a+c)}{(-a+b) c^{2}} & \frac{(b+c)(a b-a c+b c)}{(a-b) c^{2}} \\
0 & \frac{-b^{2}(b+c)}{(a-b) c^{2}} & 1+\frac{-b^{2}(b+c)}{(a-b) c^{2}}
\end{array}\right),
\end{aligned}
$$

where

$$
\begin{aligned}
& m=a c^{2}+b^{2} d, \\
& n=c^{2}+b d, \\
& p=a b c^{4}-b^{2} c^{4}+a c^{5}-b c^{5}+a b^{3} c d+a b^{3} d^{2}, \\
& q=a b c^{3}+a c^{4}-b c^{4}+a b^{2} c d+a b c^{2} d+a b^{2} d^{2} .
\end{aligned}
$$

Suppose to get contradiction that $\varphi_{4}$ is reducible. Then there exists a proper nonzero invariant subspace $S$, where 
the dimension of $S$ is either 1 or 2 . We will show that a contradiction is obtained in each of the following cases.

1. Assume that the dimension of $S$ is one. From the diagonal matrix, $A_{12}$, we see that the subspace $S$ has to be generated by $e_{1}+u e_{2}, e_{2}$ or $e_{3}$, where $\mathrm{u}$ is a complex number.

\section{Case 1.}

$S=<e_{1}+u e_{2}>$. Since $e_{1}+u e_{2} \in S$, it follows that $A_{23}\left(e_{1}+u e_{2}\right) \in S$, which implies that $(b+c) u=0$. We have to consider the case $b+c=0$ and the case $u=0$.

(a) Let $b+c=0$.

- If $u \neq 0$, then $A_{13}\left(e_{1}+u e_{2}\right) \notin S$, a contradiction.

- If $u=0$, then $A_{34} e_{1} \in S$ and $A_{14} e_{1} \in S$. This implies that $a+b=0$, a contradiction.

(b) Let $u=0$.

- If $c+d \neq 0$, then $A_{34} e_{1} \notin S$, a contradiction.

- If $c+d=0$ and $b-c \neq 0$, then $A_{24} e_{1} \notin S$, a contradiction.

- If $c+d=0$ and $b-c=0$, then $A_{14} e_{1} \notin S$, a contradiction.

\section{Case 2.}

$S=<e_{2}>$. Since $e_{2} \in S$, it follows that $A_{23} e_{2} \in S$ which implies that $b+c=0$. If $b+c=0$, then $A_{13} e_{2} \notin S$, a contradiction.

\section{Case 3.}

$S=<e_{3}>$. Since $e_{3} \in S$, it follows that $A_{23} e_{3} \in S$ which implies that $b+c=0$. If $b+c=0$, then $A_{13} e_{3} \notin S$, a contradiction.

2. Assume that the dimension of $S$ is two. We consider the cases $<e_{2}, e_{3}>$ and $\left.<e_{1}+u e_{2}, e_{3}\right\rangle$.

\section{Case 4.}

$S=<e_{2}, e_{3}>$. The proof goes along exactly same lines as

Case 2.

\section{Case 5.}

$S=<e_{1}+u e_{2}, e_{3}>$. Since $e_{1}+u e_{2} \in S$, it follows that $A_{23}\left(e_{1}+u e_{2}\right) \in S$. This implies that $(c+b)\left(u-\frac{a b-a c+b c}{a b}\right) u=0$.

(a) Let $c+b=0$.

- If $u \neq 0$ and $u \neq \frac{a^{2}+a b-b^{2}}{a^{2}}$, then $A_{13}\left(e_{1}+u e_{2}\right) \notin S$, a contradiction .
- If $u=0$ and $b-d \neq 0$, then $A_{34} e_{1} \notin S, \quad a$ contradiction .

- If $u=0$ and $b-d=0$, then $A_{24} e_{1} \notin S, \quad a$ contradiction.

- If $u=\frac{a^{2}+a b-b^{2}}{a^{2}}$, then $A_{13} e_{3} \notin S$, a contradiction.

(b) Let $u=\frac{a b-a c+b c}{a b}$.

- If $d \neq \frac{-a c^{2}}{b^{2}}$ and $d \neq \frac{a c}{b}$, then $\mathrm{A}_{14} e_{3} \notin S$, a contradiction.

- If $d=\frac{-a c^{2}}{b^{2}}$ and $a^{2}+a b+b c \neq 0$, then $A_{24} e_{3} \notin S$, a contradiction .

- If $d=\frac{-a c^{2}}{b^{2}} \quad$ and $\quad a^{2}+a b+b c=0$, then $A_{24}\left(e_{1}+u e_{2}\right) \notin S$, a contradiction.

- If $d=\frac{a c}{b}$ and $c \neq b$, then $A_{34}\left(e_{1}+u e_{2}\right) \notin S$, a contradiction.

- If $d=\frac{a c}{b}$ and $c=b$ and $b \neq \frac{-a}{2}$, then $A_{24}\left(e_{1}+u e_{2}\right) \notin S$, a contradiction.

- If $d=\frac{a c}{b}$ and $c=b=\frac{-a}{2}$, then $A_{13}\left(e_{1}+u e_{2}\right) \notin S$, a contradiction .

(c) Let $u=0$.

If $c+d \neq 0$ then $A_{34} e_{1} \notin S$, a contradiction.

If $c+d=0$ and $b-c \neq 0$, then $A_{24} e_{1} \notin S, \quad a$ contradiction .

If $c+d=0$ and $b-c=0$, then $A_{14} e_{1} \notin S, \quad a$ contradiction .

Almost the same proof, as in the case $a^{2} \neq b^{2}$, is applied to each of the cases $b^{2} \neq c^{2}$ and $c^{2} \neq d^{2}$. In each of these cases, we conjugate the corresponding representation by the invertible matrices

$N=\left(\begin{array}{ccc}0 & 1 & 1 \\ 0 & 0 & 1-b^{-1} c \\ 1 & 0 & 1\end{array}\right)$ and $P=\left(\begin{array}{ccc}0 & 1 & 0 \\ 1 & 0 & c d^{-1} \\ 1 & 0 & 1\end{array}\right)$

respectively.

\section{Lemma 5.}

For $(a, b, c, d) \in\left(\mathbb{C}^{*}\right)^{4}$, the reduced Wada's represntation $\varphi_{4}(a, b, c, d): P_{4} \rightarrow G L_{3}(\mathbb{C})$ is reducible if $a^{2}=b^{2}=c^{2}$ $=d^{2}$. 
Proof. It is clear that we have $2^{3}$ cases. They are
(1) $-a=+b=+c=+d$
(5) $+a=+b=-c=-d$
(2) $+a=-b=+c=+d$
(6) $+a=-b=+c=-d$
(3) $+a=+b=-c=+d$
(7) $+a=-b=-c=+d$
(4) $+a=+b=+c=-d$
(8) $+a=+b=+c=+d$.

Under each condition, we find a proper nonzero invariant subspace of the complex specialization of the reduced Wada's representation $\varphi_{4}$. The subspaces for (1), (2), (3), (4), (5), (6), (7) and (8) are $\left\langle e_{1}\right\rangle,\left\langle e_{1}+e_{2}+e_{3}\right\rangle,\left\langle e_{2}\right\rangle$, $\left\langle e_{3}\right\rangle, \quad\left\langle e_{2}+e_{3}\right\rangle, \quad\left\langle e_{1}+e_{2}\right\rangle, \quad\left\langle e_{1}+e_{3}\right\rangle$ and $<e_{1}-e_{2}, e_{1}+e_{3}>$, respectively.

Combining Lemma 4 and Lemma 5, we get our main theorem:

\section{Theorem 6.}

For $(a, b, c, d) \in\left(\mathbb{C}^{*}\right)^{4}$, the reduced Wada's represntation $\varphi_{4}(a, b, c, d): P_{4} \rightarrow G L_{3}(\mathbb{C})$ is reducible if and only if $a^{2}=b^{2}=c^{2}=d^{2}$.

\section{ACKNOWLEDGEMENTS}

None declared.

\section{CONFLICT OF INTERESTS}

None declared.

\section{REFERENCES}

[1] Abdulrahim MN. Wada's Representation is of Burau Type. J Algebra Comput 2001; 17(3):1-9.

[2] Birman JS. Braids, links and mapping class groups. annals of mathematical studies. vol. 82. New Jersey: Princeton University Press 1975.

[3] Hansen VL. Braids and Coverings. London Math. Soc., Cambridge: Cambridge University Press 1989. 